\title{
A Novel Emulsion Adjuvant Increases the Immunogenic Responses and Protective Efficacy of an Inactivated Influenza Vaccine in BALB/c Mice
}

\author{
Shaobin Wang ${ }^{a}$ Chang-Wen Ke ${ }^{b}$ Li-Rong Zou ${ }^{b}$ Zhang Jing ${ }^{c}$ Guo-Xiu Xie ${ }^{a}$ \\ Xiaoman $\mathrm{Li}^{\mathrm{a}}$ \\ ${ }^{a}$ College of Life Science, Hunan Normal University, Changsha, ${ }^{b}$ Center for Disease Control and Prevention of \\ Guangdong Province (GDCDC), Guangzhou, and ' Institute of Zoology, Chinese Academy of Sciences, \\ Beijing, China
}

Key Words

Oil emulsion $\cdot$ APR8 vaccine $\cdot$ MF59 $\cdot$ WAF3 $\cdot$ Adjuvant

\begin{abstract}
Objective: The research of adjuvants is a hot topic in vaccinology. In this paper, we were seeking a concise method to produce a stable and effective emulsion adjuvant. Methods: A novel emulsion [Well Adjuvant Formulation 3 (WAF3)] was produced by adding additional glycerol and using a timely cooling process. Surface morphology analysis of the novel emulsion was performed by atomic force microscopy. Animal experimentation was used to evaluate the efficacy of the novel adjuvant. Results: Surface morphology analysis of the novel emulsion showed a homogeneous distribution of the antigen protein. We also confirmed the morphological changes of the WAF3-carrying antigen. The WAF3-adjuvanted vaccine engendered higher antibody responses 128 -fold compared to that of a naked antigen. Furthermore, in the presence of the WAF3 adjuvant, the influenza virus antigen was able to reduce the titer disparities of IgG2a and IgG1. The WAF3-adjuvanted influenza vaccine was able to provide full protection and alleviation of infective symptoms as other clinically adjuvanted vaccines. Conclusion: The WAF3 adjuvant is a promising candidate to be further investigated in the development of emulsion adjuvants.
\end{abstract}

Copyright $\odot 2009$ S. Karger AG, Basel

\section{KARGER}

Fax +4161306 1234 E-Mail karger@karger.ch www.karger.com
(C) 2009 S. Karger AG, Basel

0300-5526/09/0525-0252\$26.00/0

Accessible online at:

www.karger.com/int

\section{Introduction}

The idea of using certain materials to enhance immune responses has been recognized for many years [1]. Since the discovery of aluminum hydroxide as an adjuvant for tetanus toxoids, a lot of components were investigated for their adjuvanticity [2]. Preclinical and clinical studies have confirmed that adjuvants can offer the opportunity to enhance vaccine efficacy and overcome impaired immune responses induced by conventional protein vaccines $[3,4]$.

Emulsion adjuvants are important parts of adjuvant categories [5]. Emulsions are known as effective adjuvants, able to generate high and durable antibody responses for protein vaccines [6, 7]. In 1916, oil emulsion was used in vaccine formulation by Moignic and Pinoy [8]. After decades, Freund et al. developed Freund's incomplete and complete adjuvants [9]. Freund's complete adjuvant cannot be used for humans because of severe side effects, including pain, abscess formation and fever. Subsequent reports explained that these side effects were related to the bacterial component of Freund's complete adjuvant [5]. Freund's incomplete adjuvant lacks the bacterial component and has been applied in several animal vaccines. However, as the mineral oil used in it can cause tumors in mice, Freund's incomplete adjuvant cannot be used for human vaccines. In the 1980s, a potent delivery

E-Mail home-alone2008@hotmail.com 
system (Syntex adjuvant formulation) was developed using a biodegradable oil (squalene), whose side effects were acceptable for human use [6]. However, because of the addition of a muramyl dipeptide derivative and pluronic polymer surfactant (L121), the Syntex adjuvant formulation was not used in humans [10].

Subsequently, the Chiron Corporation developed a squalene emulsion (MF59) without additional immunostimulators. MF59 emulsion is a typical oil-in-water emulsion system, which contains $5 \%$ squalene, $0.5 \%$ Tween 80 , and $0.5 \%$ Span 85 , with a homogeneous droplet size and low viscosity [11]. It has been proven that MF59 emulsion is a potent adjuvant with an optimal safety profile for various vaccines [12]. MF59 enhances the immunogenicity of influenza vaccines and has been shown to be a more potent adjuvant than alum for hepatitis B vaccines in baboons [3]. To date, the MF59-adjuvanted influenza vaccine is licensed in more than 20 countries [13]. The vaccination population of the MF59adjuvanted flu vaccine consists of more than 30 million subjects [14].

Nevertheless, there are some questions which limit this promising potentiator. First, a homogenizer and microfluidizer are always required during the preparation of MF59. In other words, the preparation method is very tedious. Moreover, large droplets should be removed by a $0.22-\mu \mathrm{m}$ filter $[15,16]$. In addition, MF59 emulsion is a Th2-directing adjuvant for protein vaccine. It feebly augments Th1 immune responses $[15,17]$. Therefore, it is much more valuable to develop concise preparation methods and emulsion systems which have the potential to be used with a variety of protein antigens.

In this paper, we prepared a novel emulsion adjuvant according to a much more concise method, named 'Well Adjuvant Formulation 3' (WAF3). Then, we analyzed the surface morphology of WAF3 microspheres by atomic force microscopy (AFM). We also compared the immunogenic responses of an inactivated influenza vaccine in combination with a WAF3 adjuvant and evaluated the protective efficacy of WAF3 emulsion in mice.

\section{Materials and Methods}

Mice

Inbred female $\mathrm{BALB} / \mathrm{c}$ mice, 6-8 weeks old, were purchased from the Laboratory Animal Center, Hubei Academy of Medical Sciences, and raised in specific pathogen-free living quarters of laboratory animals at the College of Life Science, Hunan Normal University.
Preparation of the WAF3 Emulsion

The WAF3 emulsion was prepared as follows. Glycerol $(20 \%$, $\mathrm{v} / \mathrm{v})$, squalene $(5 \%, \mathrm{v} / \mathrm{v})$, Tween $80(0.2 \%, \mathrm{v} / \mathrm{v})$ and Span $85(1 \%$, $\mathrm{v} / \mathrm{v}$ ) were emulsified by a vortex agitator for $5 \mathrm{~min}$. Then, the coarse emulsion was submerged in liquid nitrogen for $30 \mathrm{~s}$ and dissolved in an ice bath. The dissolved mixture was mixed with a syringe. Meanwhile, phosphate-buffered saline (PBS) including the inactivated antigen was added as an aqueous phase. Finally, the oil phase and aqueous phase were mixed slightly to produce the stable emulsion.

\section{The Surface Morphology of WAF3 Droplets}

Surface topography of emulsion droplets (i.e., roughness) can also affect the type, quantity and conformation of an adsorbed protein layer. In order to investigate the surface roughness of the new emulsion surfaces, AFM was used. WAF3 microspheres were prepared as specified above and examined by an atomic force microscope (SAP3800N, SPI, Seiko Corporation, Tokyo, Japan) in the tapping mode. The topography images were further processed by second-order line flattening and the images were exported to Image (NIH software, USA).

\section{Preparation of the MF59 Emulsion}

The MF59 emulsion was prepared according to Ott et al. [15]. Briefly, Tween 80 was dissolved in a PBS buffer solution. Separately, Span 85 was dissolved in squalene. These 2 solutions were mixed and processed to obtain a coarse emulsion. Then, this coarse emulsion was passed through a microfluidizer to yield the stable emulsion. Finally, the bulk emulsion was filtered through a $0.22-\mu \mathrm{m}$ filter to remove large droplets.

\section{Immunization and Specimens}

An inactivated influenza virus A/PR/8/34 (H1N1) vaccine was prepared by the Shanghai Institute of Biological Products and the concentration was checked by a BCA kit (Pierce, Thermo Fisher Scientific Inc., Portsmouth, N.H., USA). The MF59-adjuvanted vaccine was prepared by mixing MF59 1:1 with an inactivated antigen. Each mouse dose contained a $0.015-\mu \mathrm{g}$ antigenic protein. The inactivated virus was adsorbed to aluminum hydroxide at $4^{\circ}$. Each mouse dose contained $100 \mu \mathrm{g}$ of alum. Animals were immunized i.p. twice, 3 weeks apart, at a volume of $200 \mu l$. The control mice were injected i.p. with $200 \mu$ l PBS. Three weeks after primary immunization and 1 week after secondary immunization, blood samples were collected from the tail vein of the immunized mice.

\section{ELISA for Serum Antibodies}

The titers of IgG and IgG isotypes were measured against the inactivated influenza virus PR8 vaccine by ELISA, as previously described [18]. ELISA was performed sequentially from the solid phase polystyrene plate with a ladder of reagents consisting of: 1 st, an inactivated influenza virus PR8 vaccine; 2 nd, 2 -fold diluted serum; 3rd, biotin-labeled goat anti-mouse IgG (Southern Biotechnology Associates Inc., Birmingham, Ala., USA); 4th, streptavidin conjugated with alkaline phosphatase (Southern Biotechnology Associates Inc.); and finally, $p$-nitrophenyl-phosphate. The chromogen count was measured for absorbance at 414 and $405 \mathrm{~nm}$ in an autoreader (Labsystems Muliskan Ascent, Thermo Fisher Scientific Inc., Portsmounth, N.H., USA). 


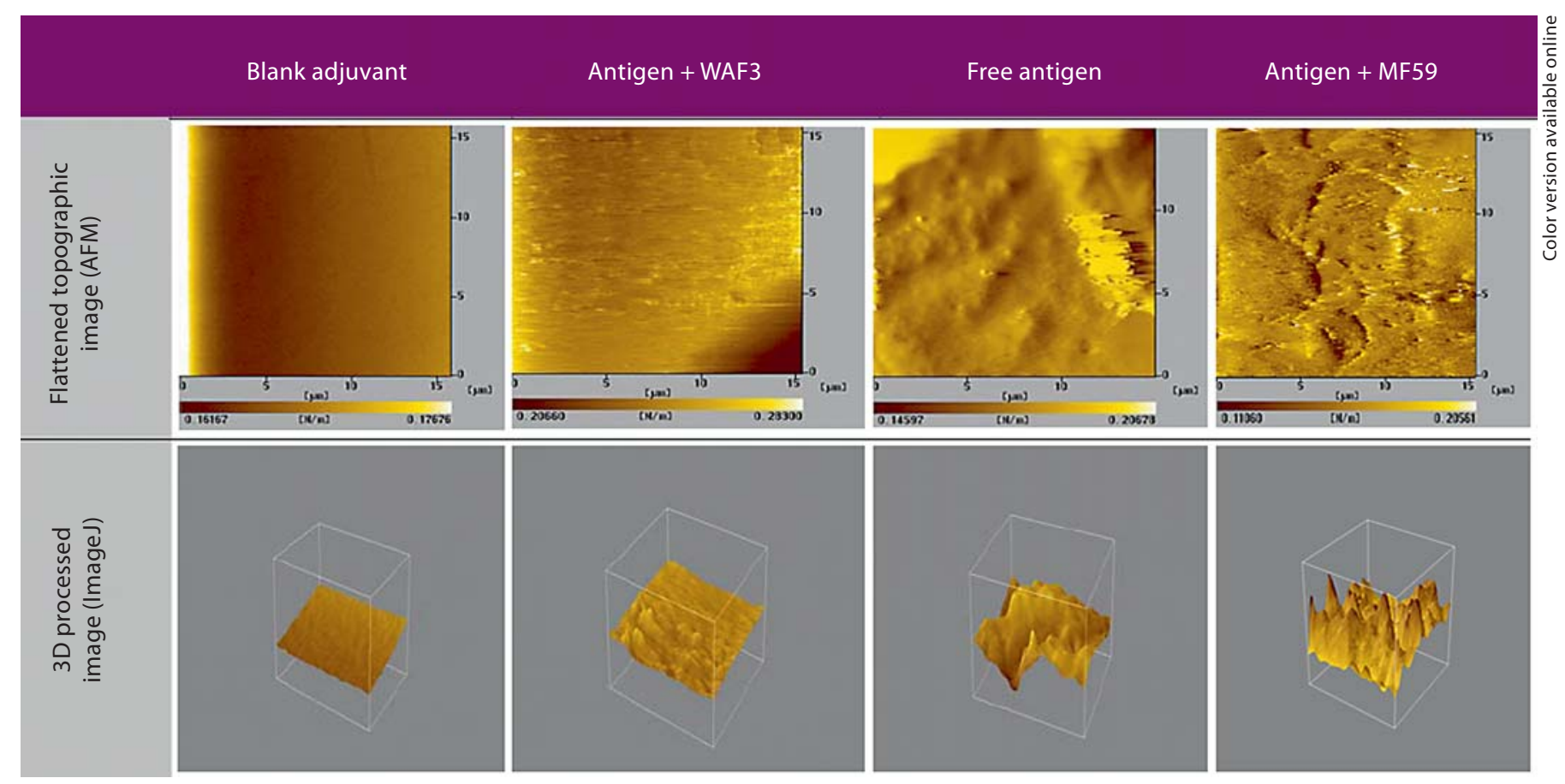

Fig. 1. AFM topography of various WAF3 emulsion surfaces.

Table 1. Root mean square roughness values $( \pm S D)$ obtained from the average of 3 images of WAF3 emulsion surfaces

\begin{tabular}{ll}
\hline WAF3 emulsions & Root mean square roughness \\
\hline Blank WAF3 emulsion & $109.34 \pm 10.88$ \\
Antigen-carrying emulsion & $127.41 \pm 15.12$ \\
Free antigen & $127.59 \pm 17.03$ \\
Antigen + MF59 emulsion & $130.09 \pm 18.66$
\end{tabular}

Virus Infection

Two weeks after the 2 nd immunization, each mouse was anesthetized and challenged with $20 \mu \mathrm{l}$ of homologous A/PR/8/34 $(\mathrm{H} 1 \mathrm{~N} 1)$ virus suspension containing a $40 \times 50 \%$ lethal dose by intranasal drip. Changes of body weight and the survival rate of the infected mice were observed to evaluate the protective efficacy of these adjuvanted vaccines.

\section{Results}

\section{Surface Characteristics of the WAF3 Emulsion}

The WAF3-adjuvanted vaccine was analyzed by means of AFM to determine the characteristics of the
WAF3 emulsion as well as the interplay between antigens and emulsion microspheres. To characterize changes in surface roughness, AFM images were taken of a blank WAF3 emulsion, an antigen-coated emulsion and a free antigen. Images were processed using ImageJ (NIH Software), which provided a flattened topographic image and a 3-dimensional image. Figure 1 shows the captured images of the emulsion and antigen samples. ImageJ was also used to generate root mean square roughness. Table 1 lists the average root mean square roughness values obtained for the various WAF3 emulsion surfaces.

\section{Immunogenic Responses to WAF3-Adjuvanted \\ Vaccine}

To investigate the suitability of the antigen-loaded WAF3 emulsion, we compared the serum responses of mice after 2 i.p. vaccinations with various vaccines. After the booster immunization, the WAF3-adjuvanted vaccine generated stronger serum IgG titers than the other groups $(\mathrm{p}<0.05)$. We also evaluated the blood samples collected before the secondary immunization. Interestingly, although the WAF3 emulsion enhanced the magnitude of the humoral responses at the early stage, there were no significant differences between the WAF3 group 

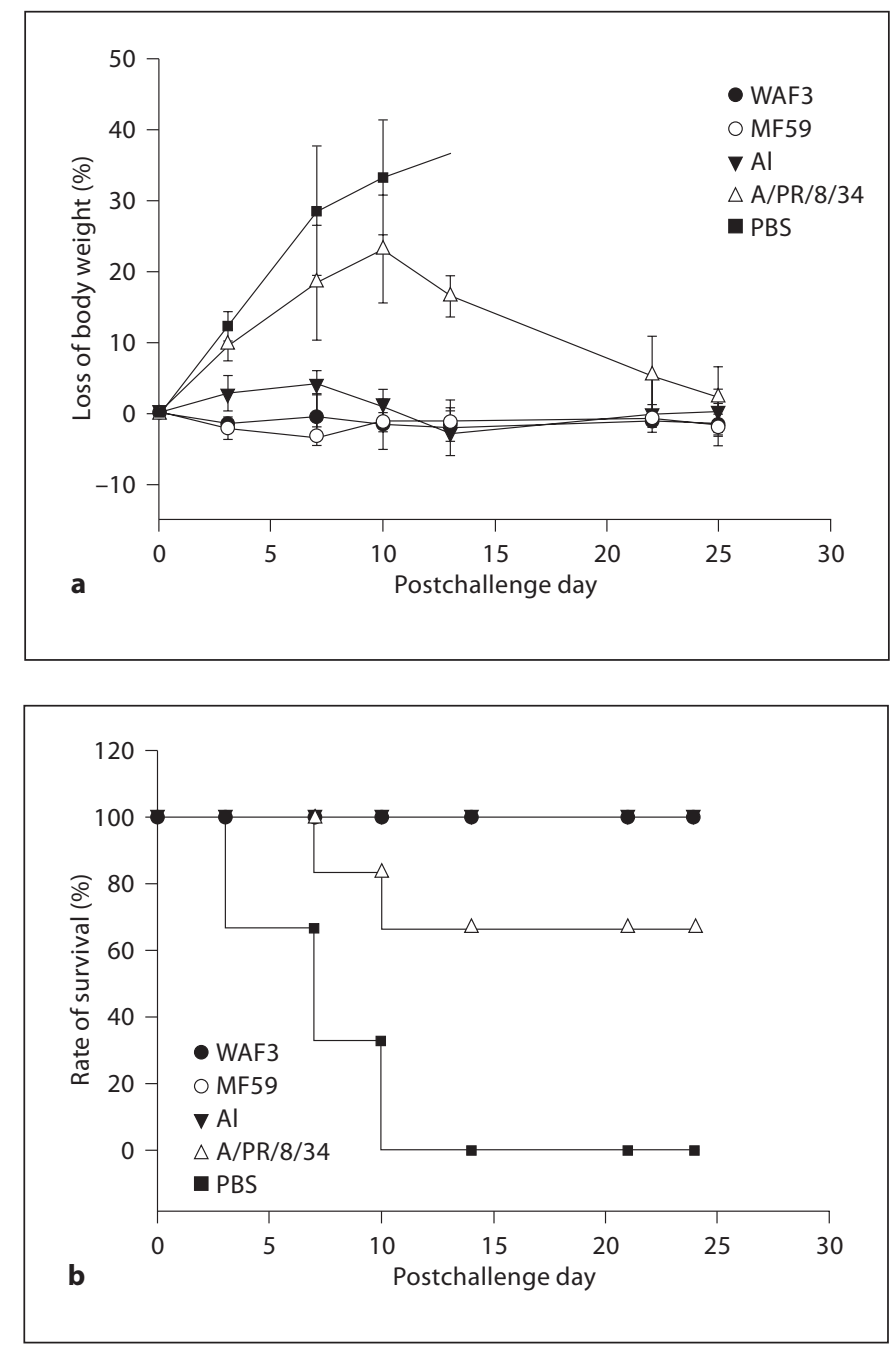

Fig. 2. Protection from adjuvanted vaccines against the lethal challenge infection in BALB/c mice. a Loss of body weight after lethal challenge. After the 2nd immunization, naive or immunized mice were intranasally infected with a $40 \times 50 \%$ lethal dose of homologous virus. Mice were monitored for changes in body weight daily for 25 days. $\mathbf{b}$ Survival rates; all mice in the PBS group died by day 10 , postchallenge.

and the MF59 group. In other words, the excellent potency of the WAF3-adjuvanted vaccine required secondary inoculation. Furthermore, we described the IgG subtype profiles of the vaccinated groups (table 2). A booster vaccination with the WAF3-adjuvanted vaccine induced stronger IgG2a or IgG1 titers as compared to other groups. Results also confirmed MF59 emulsion as a Th2-type adjuvant. Both the WAF3 and the MF59 emulsions can induce higher IgG1 titers as compared to the naked in-
Table 2. Influenza-specific serum immunoglobulin G (IgG) and IgG subtype antibody titers induced after 1 or 2 doses of the A/ $\mathrm{PR} / 8 / 34(\mathrm{H} 1 \mathrm{~N} 1)$ vaccine $^{1}$

\begin{tabular}{|c|c|c|c|c|c|}
\hline \multirow{3}{*}{$\begin{array}{l}\text { Formula- } \\
\text { tions }{ }^{2}\end{array}$} & \multirow{3}{*}{$\begin{array}{l}\text { Dose of } \\
\text { vaccine } \\
\mu \mathrm{g} / \text { dose }\end{array}$} & \multicolumn{4}{|c|}{ Serum IgG/IgG2a/IgG1 titers; $\operatorname{ELISA}\left(2^{\mathrm{n}}\right)^{3}$} \\
\hline & & \multirow{2}{*}{$\frac{\text { primary }}{\operatorname{IgG}}$} & \multicolumn{3}{|l|}{ secondary } \\
\hline & & & $\operatorname{IgG}$ & $\operatorname{IgG} 2 \mathrm{a}$ & $\operatorname{IgG} 1$ \\
\hline WAF3 & 0.015 & $15.0 \pm 1.0$ & $20.7 \pm 1.3^{*}$ & $18.0 \pm 0.9^{*}$ & $18.3 \pm 0.6^{*}$ \\
\hline MF59 & 0.015 & $14.0 \pm 1.0$ & $17.3 \pm 0.5$ & $14.7 \pm 0.8$ & $16.7 \pm 1.0$ \\
\hline $\mathrm{Al}(\mathrm{OH})_{3}$ & 0.015 & $13.7 \pm 0.9$ & $15.7 \pm 0.6$ & $12.3 \pm 1.0$ & $10.7 \pm 0.6$ \\
\hline PR8 & 0.015 & $11.7 \pm 0.6$ & $13.7 \pm 1.0$ & $11.3 \pm 1.5$ & $9.0 \pm 1.5$ \\
\hline PBS & - & 0 & 0 & 0 & 0 \\
\hline
\end{tabular}

${ }^{*} \mathrm{p}<0.05$ between the MF59 group and the WAF3 group.

${ }^{1}$ Female mice were immunized twice, 3 weeks apart, with various adjuvanted whole virus vaccines. Serum samples from the animals were collected 3 weeks after primary immunization and 1 week after the booster. Serum antibody titers were measured by ELISA.

${ }^{2}$ PR8 + WAF3 = inactivated influenza PR8 virus + WAF3 emulsion; PR8 + MF59 = inactivated influenza PR8 virus + MF59 emulsion; $\mathrm{PR} 8+\mathrm{Al}(\mathrm{OH})_{3}=$ inactivated influenza PR8 virus + $\mathrm{Al}(\mathrm{OH})_{3} ; \mathrm{PR} 8=$ immunization with inactivated influenza PR8 virus alone.

${ }^{3}$ Serum samples were diluted 2-fold serially, and 'n' represents the dilution factor. Values represent mean $\pm \mathrm{SD}$ for each group.

activated vaccine. Another investigation by our group showed that all the adjuvants can reduce the IgG2a/IgG1 ratio of the whole virus vaccine. The IgG2a/IgG1 ratio decreased in the order of alum-absorbed vaccine, MF59adjuvanted vaccine and WAF3-adjuvanted vaccine (data not shown).

\section{Protective Quality of the WAF3-Adjuvanted Vaccine}

To evaluate the protective quality of the WAF3-adjuvanted vaccine against the lethal challenge in the mouse model, body weight changes and survival rates were monitored after the homologous virus challenge (fig. 2a, b). The blank group (injected with PBS) with the challenge infection showed a significant and increased loss in body weight and symptoms of severe influenza virus infection, such as ruffled fur and shiver. In the end, all mice in the blank group lost 35\% of their body weight and died. The body weight loss of the antigen-alone group was much less severe than that of the blank group. However, the survival rate of the antigen-alone group was $67.7 \%$. In contrast, there were no symptoms detected in the adjuvanted groups. 


\section{Discussion}

In the present study, we investigated a method of preparing emulsion adjuvant and prepared a novel emulsion adjuvant (WAF3). Results demonstrate that the WAF3 emulsion holds great promise as a delivery system for protein vaccines. Measurement results obtained by AFM showed that the roughness of the blank WAF3 emulsion microspheres was very indistinct. Cavities and pores were not detected on the surface of the emulsion droplets, which are often seen on the surface of polymer microspheres [19]. In contrast, the surface of the load-carrying WAF3 emulsion microspheres was rather rough. Clearly, there were lots of flossy proteins adsorbed on the surface of the WAF3 emulsion microspheres. Otherwise, the adsorbed antigen protein is much less compact than unrestricted proteins dissolved in PBS (fig. 1). Moreover, conformational changes were detected around the adsorbed antigens. This is likely to be important for their excellent adjuvanticity. First of all, these adsorbed formulations can help protein vaccines to flow into a targeting position with a higher local concentration where the macrophages are enriched by the emulsion adjuvant [20]. Secondly, the surface restructuring of the adsorbed antigens is necessary for their recognition by receptor cells [21, 22]. The apparent relationship between the exposed area of a protein and antigenic reactivity has been previously confirmed. Moreover, the adsorption of an antigen could induce a surface rearrangement of the protein, resulting in the unmasking of cryptic epitopes, which is important for the specificity of immune responses [23].

Immunogenic analysis of the WAF3-adjuvanted vaccine indicated that a booster inoculation of the WAF3- adjuvanted vaccine can induce stronger humoral immune responses than the immunization with antigen alone. However, the superior adjuvanticity of the WAF3 emulsion seems to depend on a repeated injection. We surmised that the booster-requiring characteristic is allied to the adsorbed formulations and the incompact state of the adjuvanted antigens, but this remains to be proven. IgG subtype profiles of the WAF3-adjuvanted vaccine indicated that the WAF3 emulsion induced higher IgG2a/ IgG1 titers than other groups. It is well known that IgG2a and IgG1 antibodies perform different obligations in mice [24]. IgG2a is accomplished in complement activation and antibody-dependent cellular immunity. Thus, IgG2a is more effective in preventing intracellular virus replication. On the other hand, IgG1 plays a role in neutralizing viruses and in reducing the release of a mature virus [25]. WAF3 emulsion did not change the subtype profiles of the protein vaccine, but was able to markedly enhance both the IgG2a and IgG1 production. This is the groundwork of the excellent protective quality of the adjuvanted vaccine. The WAF3-adjuvanted group consistently attained $100 \%$ survival rates with lower loss of body weight. This may be related to higher IgG2a titers, which participates in virus clearance via antibody-mediated cellular immunity.

In conclusion, the present study demonstrates that the WAF3 emulsion is a potent 'ready to formulate' adjuvant formulation for the inactivated flu vaccine. It was manufactured in a simple protocol. With a much lower concentration of antigens, the WAF3-adjuvanted flu vaccine can provide optimal protection against lethal virus infection in mouse model. Studies to evaluate these WAF3 emulsions with other antigens are currently underway.

\section{References}

1 Glenny AT, Pope CG, Waddington H, Wallace V: The antigenic value of toxoid precipitated by potassium alum. J Pathol Bacteriol 1926;29:38-45.

2 Aguilar JC, Rodriguez EG: Vaccine adjuvants revisited. Vaccine 2007;25:3752-3762.

-3 Traquina P, Morandi M, Contorni M, Van Nest G: MF59 adjuvant enhances the antibody response to recombinant hepatitis $B$ surface antigen vaccine in primates. J Infect Dis 1996;174:1168-1175.

4 Alving CR: Design and selection of vaccine adjuvants: animal models and human trials. Vaccine 2002;20(suppl 3):S56-S64.
5 Murray RP, Cohen P, Hardegree MC: Mineral oil adjuvants: biological and chemical studies. Ann Allergy 1972;30:146-151.

6 6 Hjorth RN, Bonde GM, Piner ED, Goldberg KM, Levner MH: The effect of Syntex adjuvant formulation (SAF-m) on humoral immunity to the influenza virus in the mouse. Vaccine 1997;15:541-546.

7 Kang ML, Jiang HL, Kang SG, Guo DD, Lee DY, Cho CS, Yoo HS: Pluronic F127 enhances the effect as an adjuvant of chitosan microspheres in the intranasal delivery of Bordetella bronchiseptica antigens containing dermonecrotoxin. Vaccine 2007;25:46024610 .
8 Moignic L, Pinoy: Les vaccins en emulsion dans les corps gras ou 'lipo-vaccins.' Comptes Rendus de la Société de Biologie 1916;79: 201-203.

-9 Freund J, Casals J, Hosmer EP: Sensitization and antibody formation after injection of tubercle bacilli and paraffin oil. Proc Soc Exp Biol Med 1937;37:509-513.

10 McElrath MJ: Selection of potent immunological adjuvants for vaccine construction. Semin Cancer Biol 1995;6:375-385.

11 Ott G, Barchfeld GL, Van Nest G: Enhancement of humoral response against human influenza vaccine with the simple submicron oil/water emulsion adjuvant MF59. Vaccine 1995; 13:1557-1562. 
$>12$ Ott G, Barchfeld GL, Chernoff D, Radhakrishnan R, van Hoogevest P, Van Nest G: MF59. Design and evaluation of a safe and potent adjuvant for human vaccines. Pharm Biotechnol 1995;6:277-296.

-13 Puig Barbera J, Gonzalez Vidal D: MF59-ad juvanted subunit influenza vaccine: an improved interpandemic influenza vaccine for vulnerable populations. Expert Rev Vaccines 2007;6:659-665.

14 O’Hagan DT, Wack A, Podda A: MF59 is a safe and potent vaccine adjuvant for flu vaccines in humans: what did we learn during its development? Clin Pharmacol Ther 2007; 82:740-744.

15 Ott G, Radhakrishnan J, Fang JF, Hora M: The adjuvant MF59: a 10-year perspective; in O'Hagan DT (ed): Vaccine Adjuvants: Preparation Methods and Research Protocols. Totowa, Humana Press, 2000, pp 211-228.

-16 Valensi JP, Carlson JR, Van Nest GA: Systemic cytokine profiles in BALB/c mice immunized with trivalent influenza vaccine containing MF59 oil emulsion and other advanced adjuvants. J Immunol 1994;153: 4029-4039.
17 Ott G, Singh M, Kazzaz J, Briones M, Soenawan E, Ugozzoli M, O'Hagan DT: A cationic sub-micron emulsion (MF59/DOTAP) is an effective delivery system for DNA vaccines. J Control Release 2002;79:1-5.

18 Chen Z, Sahashi Y, Matsuo K, Asanuma H, Takahashi H, Iwasaki T, Suzuki Y, Aizawa C, Kurata T, Tamura S: Comparison of the ability of viral protein-expressing plasmid DNAs to protect against influenza. Vaccine 1998 . 16:1544-1549.

19 Bouillot P, Ubrich N, Sommer F, Duc TM, Loeffler JP, Dellacherie E: Protein encapsulation in biodegradable amphiphilic microspheres. Int J Pharm 1999;181:159-172.

20 Dupuis M, Murphy TJ, Higgins D, Ugozzoli M, Van Nest G, Ott G, McDonald DM: Dendritic cells internalize vaccine adjuvant after intramuscular injection. Cell Immunol 1998, 186:18-27.
21 Roguin LP, Retegui LA: Monoclonal antibodies inducing conformational changes on the antigen molecule. Scand J Immunol 2003;58:387-394.

22 Mazza MM, Retegui LA: Monoclonal antibodies to human growth hormone induce an allosteric conformational change in the antigen. Immunology 1989;67:148-153.

23 Guillon C, Mayol K, Terrat C, Compagnon C, Primard C, Charles MH, Delair T, Munier S, Verrier B: Formulation of HIV-1 Tat and p24 antigens by PLA nanoparticles or MF59 impacts the breadth, but not the magnitude, of serum and faecal antibody responses in rabbits. Vaccine 2007;25:7491-7501.

24 Coutelier JP, van der Logt JT, Heessen FW, Warnier G, Van Snick J: IgG2a restriction of murine antibodies elicited by viral infections. J Exp Med 1987;165:64-69.

25 Hocart MJ, Mackenzie JS, Stewart GA: The immunoglobulin $G$ subclass responses of mice to influenza A virus: the effect of mouse strain, and the neutralizing abilities of individual protein A-purified subclass antibodies. J Gen Virol 1989;70:2439-2448. 